\title{
La désintermédiation des technologies: Vers SCPH 2015 et au-delà
}

\author{
par Richard Jones
}

$\mathrm{N}$ ombre d'entre vous se souviendront du grand mot qu'utilisait dans ses présentations l'ancien président de la $\mathrm{SCPH}$, Neil Johnson : la " désintermédiation ». Neil faisait référence à l'émergence des assistants numériques personnels, les ANP, comme mines de données sur les médicaments qui remplaceraient les pharmaciens, véritables " encyclopédies ambulantes d'information pharmacothérapeutique ». Son message était que les pharmaciens devaient être pleinement conscients de leur valeur dans les soins aux patients, ou alors ils risquaient l'extinction, tout comme les fabricants de glacières domestiques disparurent avec l'arrivée des réfrigérateurs.

Le milieu des soins de santé connait une incroyable explosion des technologies, notamment avec l'arrivée des outils comme les ANP qui renferment des bibliothèques d'information sur les médicaments, des dossiers médicaux électroniques, sans compter les robots qui préparent et distribuent les médicaments, et les nombreuses autres innovations toutes proches. Malgré le défi posé par le financement de ces technologies, celles-ci représentent néanmoins la meilleure occasion pour les pharmaciens de concrétiser la vision suggérée par Roy Romanow dans son rapport il y a dix ans'. Elles sont aussi essentielles à la réalisation de la vision articulée dans le projet SCPH 2015!

Imaginez un instant un système de production dans lequel les technologies de distribution remplaceraient les postes de prélèvement manuel; les technologies de préparation remplaceraient les méthodes de remplissage manuel des seringues, l'emploi des hottes à flux laminaire, voire même les hottes biologiques; les ANP et les réseaux informatiques hospitaliers rendraient la bibliothèque des sciences médicales entière, y compris les compilations et les analyses, accessible dans n'importe quel point de soins aux patients; et des technologies similaires permettraient l'accès partout au dossier médical complet du patient et à des outils d'enseignement lié à l'emploi des médicaments, à la fois pour les fournisseurs de soins de santé et pour les patients. Dans ce système de production du futur, quelle place resterait-il pour les pharmaciens et les techniciens en pharmacie?

Les pharmaciens n'auraient plus aucun lien direct avec le système de distribution, et ne seraient plus appelés à trouver les médicaments que cherche le personnel infirmier. Les techniciens en pharmacie seraient entièrement responsables du système d'exploitation des pharmacies, mettant en œuvre leurs talents pour appuyer la technologie et s'acquittant de toute activité que les technologies ne peuvent réaliser. Ils pourraient même travailler dans les unités de soins directement pour résoudre les problèmes liés à la production et à la préparation des doses de médicaments. Les pharmaciens n'auraient plus à saisir les ordonnances dans un lieu loin des patients, et ils n'auraient que rarement besoin de passer des heures à la bibliothèque à fouiller la littérature à propos d'un cas particulier afin de participer à la conception du schéma thérapeutique optimal pour le patient. Toutes les connaissances et toute l'information nécessaires aux pharmaciens seraient à leur portée et, inversement, le système de distribution fonctionnerait sans l'intervention des pharmaciens. Les pharmaciens seraient véritablement libres pour travailler dans les unités de soins et tirer pleinement profit des perspectives que présentent les soins pharmaceutiques, leur permettant ainsi à la fois d'atteindre les objectifs de SCPH 2015 et de réaliser la carrière enrichissante qu'ils imaginaient lorsqu'ils ont reçu leur diplôme de pharmacien.

Ce prodigieux bond en avant se traduirait ultimement par des améliorations considérables et mesurables de la qualité des soins et de l'emploi sûr des médicaments. Il ne peut y avoir de meilleures raisons de saisir l'occasion offerte par ces technologies que la perspective d'une véritable désintermédiation des pharmaciens de leur environnement fondé sur les aspects manuels et les produits, et du respect de la promesse faite aux patients par le truchement du serment de Maïmonide.

[Traduction par l'éditeur]

\section{Référence}

1. Commission sur l'avenir des soins de santé au Canada. Guidé par nos valeurs: l'avenir des soins de santé au Canada - Rapport final. Saskatoon (SK) : La Commission; 2002. Publié à www.hc-sc.gc.ca/hcs-sss/alt_formats/ hpb-dgps/pdf/hhr/romanow-fra.pdf. Consulté le 10 février 2009.

Richard Jones, R. Ph., B. Sc., B.S.P., est président élu et agent de liaison interne de la SCPH. 